\title{
CATTANEO-VERNOTTE EQUATION. IDENTIFICATION OF RELAXATION TIME USING EVOLUTIONARY ALGORITHMS
}

\author{
Bohdan Mochnacki ${ }^{1}$,Marek Paruch ${ }^{2}$ \\ ${ }^{1}$ Institute of Mathematics, Czestochowa University of Technology \\ Częstochowa, Poland \\ Higher School of Labour Safety Management in Katowice \\ Katowice, Poland \\ ${ }^{2}$ Institute of Computational Mechanics and Engineering, Silesian University of Technology \\ Gliwice, Poland \\ ${ }^{1}$ bohdan.mochnacki@im.pcz.pl, ${ }^{2}$ marek.paruch@polsl.pl
}

\begin{abstract}
The Cattaneo-Vernotte equation describing the heat conduction process in domain of solid body results from the generalization of the well - known Fourier law, in which the 'delay time' (relaxation time $\tau_{q}$ ) is introduced. The Cattaneo-Vernotte equation should be, among others, used in a case of microscale heat transfer analysis when the thermal processes are characterized by the extremely short duration (e.g. ultrafast laser pulse), the considerable temperature gradients and the very small dimensions (e.g. thin metal film). In the paper the problem of relaxation time identification is considered. In particular, the heat conduction process proceeding in domain of thin metal film subjected to a laser pulse is analyzed. The inverse problem solution is obtained using the evolutionary algorithms. The information concerning the time-dependent temperature distribution on the surface of metal film is assumed to be known. At the stage of numerical computations the finite difference method (FDM) is applied. In the final part of the paper the example of computations is shown.
\end{abstract}

\section{Introduction}

As is well known, the classical Fourier's law is written as follows

$$
q(x, t)=-\lambda \nabla T(x, t)
$$

where $q$ is a heat flux, $\lambda$ is a thermal conductivity.

To take into account the finite velocity of thermal wave and 'delay time' of heat flux with respect to temperature gradient, the generalized form of formula (1) should be introduced

$$
q\left(x, t+\tau_{q}\right)=-\lambda \nabla T(x, t)
$$


in which the parameter called the relaxation time $\tau_{q}$ appears. The value of this parameter is small and, as a rule, considering the macro-scale heat transfer the relaxation time can be neglected.

The other situation takes place in a case of micro-scale heat transfer problems. Then the assumption of the classical form of Fourier law is not acceptable and one should consider the generalization (2).

The aim of the considerations presented in the paper is the problem of relaxation time identification (using the evolutionary algorithms), at the same time the additional information necessary to solve the inverse problem results from the assumption that the time dependent temperature on the upper external surface of the system is known.

\section{Governing equations}

The well known macroscopic energy equation

$$
c \frac{\partial T(x, t)}{\partial t}=-\nabla \cdot \mathbf{q}(x, t)
$$

can be transformed to the micro-scale when in the place of the classical Fourier law, one introduces the following first-order approximation of formula (2)

$$
\mathbf{q}(x, t)+\tau_{q} \frac{\partial \mathbf{q}(x, t)}{\partial t}=-\lambda \nabla T(x, t)
$$

or

$$
-\mathbf{q}(x, t)=\tau_{q} \frac{\partial \mathbf{q}(x, t)}{\partial t}+\lambda \nabla T(x, t)
$$

This expression should be introduced to equation (3) and then

$$
c \frac{\partial T(x, t)}{\partial t}=\tau_{q} \frac{\partial}{\partial t}[\nabla \mathbf{q}(x, t)]+\nabla[\lambda \nabla T(x, t)]
$$

Substituting $-\nabla \mathbf{q}$ by $c(\partial T / \partial t)$ one obtains

$$
c\left[\frac{\partial T(x, t)}{\partial t}+\tau_{q} \frac{\partial^{2} T(x, t)}{\partial t^{2}}\right]=\nabla[\lambda \nabla T(x, t)]
$$

In the presence of internal heat sources within the domain considered the equation (7) takes a form 


$$
\begin{gathered}
c\left[\frac{\partial T(x, t)}{\partial t}+\tau_{q} \frac{\partial^{2} T(x, t)}{\partial t^{2}}\right]= \\
=\nabla[\lambda \nabla T(x, t)]+Q(x, t)+\tau_{q} \frac{\partial Q(x, t)}{\partial t}
\end{gathered}
$$

where $Q(x, t)$ is a capacity of internal heat sources considered. In the next part of the paper, this form of energy equation is accepted because the action of laser beam (the Neumann boundary condition on the upper surface of the metal film) is substituted by the 'artificial' internal heat source situated within the film domain (e.g. $[1,2])$.

In Figure 1 the domain considered is shown and the geometrical features of the thin metal film allow one to treat the problem as a $1 \mathrm{D}$ one. In this case the source function according to literature (e.g. [2]) is determined by the formula

$$
Q(x, t)=\sqrt{\frac{\beta}{\pi}} \frac{1-R}{t_{p} \delta} I_{0} \exp \left[-\frac{x}{\delta}-\sqrt{\beta} \frac{\left(t-2 t_{p}\right)^{2}}{t_{p}^{2}}\right]
$$

where $I_{0}$ is the laser intensity which is defined as total energy carried by a laser pulse per unit cross-section of the laser beam, $t_{p}$ is the characteristic time of laser pulse, $\delta$ is the characteristic transparent length of irradiated photons called the absorption depth, $R$ is the reflectivity of the irradiated surface and $\beta=4 \ln 2$. The local and temporary value of $Q(x, t)$ results from the distance $x$ between the surface subjected to the laser action and the point considered.

Introduction of the function $Q(x, t)$ causes that the boundary conditions given for $x=0$ (as for $x=L, L$ is the thickness of the plate) correspond to no-flux ones.

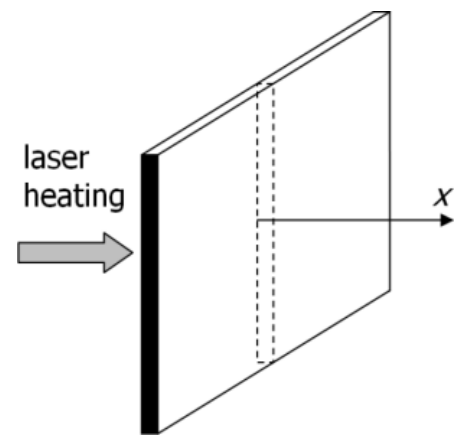

Fig. 1. Domain considered

The initial condition (initial temperature and initial heating rate) are assumed to be known. At the stage of numerical computations, the boundary-initial problem discussed has been solved using the variant of the finite difference method described in [3-5]. 


\section{Inverse problem}

To solve the inverse problem the least squares criterion is applied

$$
S\left(\tau_{q}\right)=\frac{1}{M F} \sum_{i=1}^{M} \sum_{f=1}^{F}\left(T_{i}^{f}-T_{d i}^{f}\right)^{2}
$$

where $T_{d i}^{f}$ and $T_{i}^{f}=T\left(x_{i}, t^{f}\right)$ are the measured and estimated temperatures, respectively, $M$ is the number of sensors (e.g. [6]). The minimum of functional (10) has been found using the evolutionary algorithms [7]. So, the direct problems have been solved and the results allow one to determine the 'measured' time dependent surface temperature $(x=0)$. Next, we assume that the laser parameters determining capacity of internal source function $Q(x, t)$ and also the thermal conductivity and volumetric specific heat of material are known, while the parameter $\tau_{q}$ should be determined (from a practical point of view the experimental estimation of $\tau_{q}$ is not easy).

\section{Results of computations}

In Figures 2 and 3 the example of direct problem solution is shown. The golden layer is subjected to a short-pulse laser irradiation which parameters are equal to: $R=0.93$ (reflectivity), $I_{0}=13.7 \mathrm{~J} / \mathrm{m}^{2}$ (intensity), $t_{p}=0.1 \mathrm{ps}=10^{-13} \mathrm{~s}$ (time of laser pulse), $\delta=15.3 \mathrm{~nm}$ (absorption depth). The following parameters of gold thin film are assumed: thermal conductivity $\lambda=317 \mathrm{~W} /(\mathrm{mK})$, volumetric specific heat $c=2.4897 \mathrm{MJ} /\left(\mathrm{m}^{3} \mathrm{~K}\right)$, relaxation time $\tau_{q}=8.5 \mathrm{ps}$. Initial temperature equals $T_{0}=20^{\circ} \mathrm{C}$.

Using the FDM algorithm under the assumption that $N=200$ and $\Delta t=0.005 \mathrm{ps}$ the transient temperature field has been found. In Figure 2 the temperature profiles are shown, while Figure 3 illustrates the courses of heating (cooling) curves at the points selected from the domain considered.

The identification of 'delay' time has been done using the evolutionary algorithms. The parameters of EA are collected in Table 1.

Table 1

Evolutionary algorithm parameters

\begin{tabular}{|c|c|c|c|c|c|}
\hline $\begin{array}{c}\text { Number of } \\
\text { generations }\end{array}$ & $\begin{array}{c}\text { Number of } \\
\text { chromosomes }\end{array}$ & $\begin{array}{c}\text { Prob. of } \\
\text { uniform } \\
\text { mutation }\end{array}$ & $\begin{array}{c}\text { Prob. of } \\
\text { nonuniform } \\
\text { mutation }\end{array}$ & $\begin{array}{c}\text { Prob. of } \\
\text { arithmetic } \\
\text { crossover }\end{array}$ & $\begin{array}{c}\text { Prob. of } \\
\text { cloning }\end{array}$ \\
\hline 50 & 50 & $10 \%$ & $20 \%$ & $40 \%$ & $5 \%$ \\
\hline
\end{tabular}

The final result of $\tau_{q}$ estimation corresponds to $8.499999 \cdot 10^{-12} \mathrm{ps}$, while the exact value was assumed to be $\tau_{q}=8.5 \cdot 10^{-12} \mathrm{ps}$. 


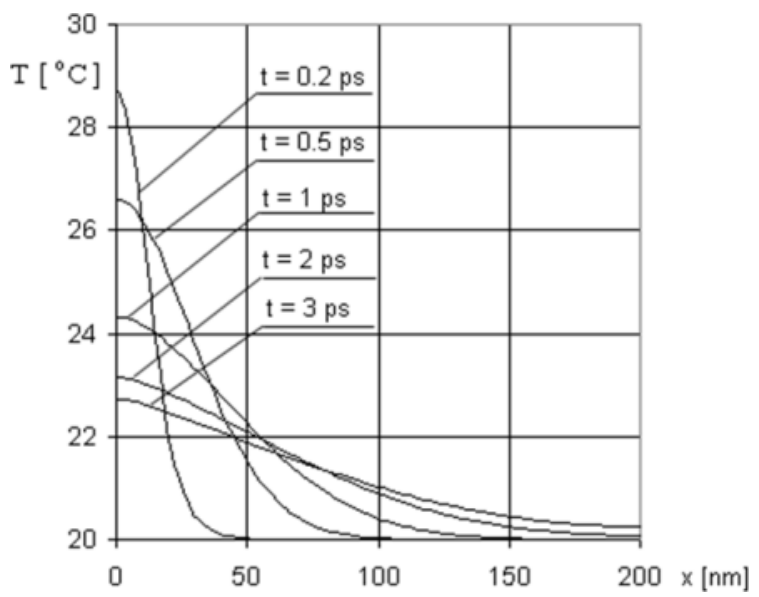

Fig. 2. Temperature profiles

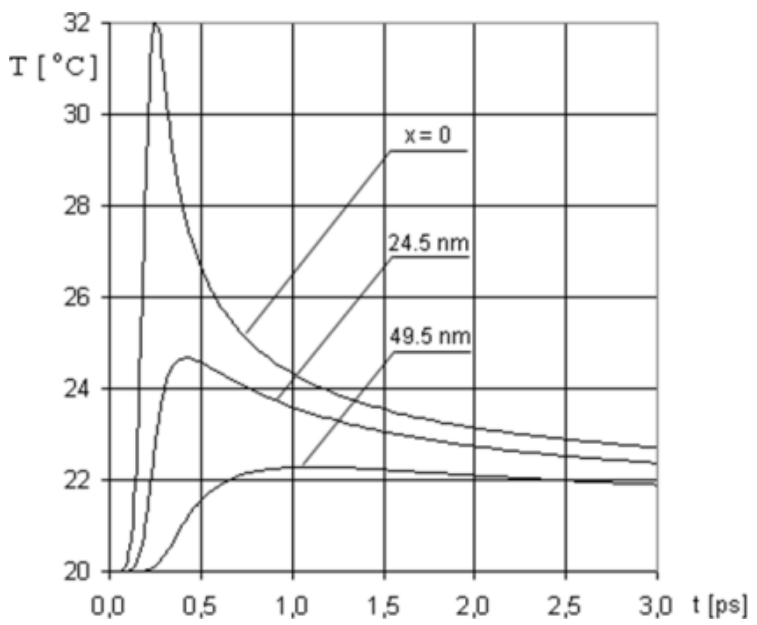

Fig. 3. Cooling (heating) curves

\section{Acknowledgement}

This work is supported by the project No. 2012/05/B/ST8/01477 sponsored by the Polish National Science Centre.

\section{References}

[1] Chen J.K., Beraun J.E., Numerical study of ultrashort laser pulse interactions with metal films, Numerical Heat Transfer 2001, Part A, 40, 1-20.

[2] Kaba I.K., Dai W., A stable three - level finite difference scheme for solving the parabolic two-step model in a 3D micro sphere heated by ultrashort-pulsed lasers, Journal of Computational and Applied Mathematics 2005, 181, 125-147. 
[3] Majchrzak E., Mochnacki B., Greer A.L., Suchy J.S., Numerical modeling of short pulse laser interactions with multi-layered thin metal films, Computer Modeling in Engineering and Sciences 2009, 41, 2, 131-146.

[4] Majchrzak E., Mochnacki B., Suchy J.S., Finite difference model of short-pulse laser interactions with thin metal film, Computer Methods in Materials Science 2009, 9, 2, 316-322.

[5] Mochnacki B., Majchrzak E., Modeling of microscale heat transfer in cylindrical domains, Computer Methods in Materials Science 2011, 11, 2, 337-342.

[6] Mochnacki B., Majchrzak E., Identification of macro and micro parameters in the solidification model, Bulletin of the Polish Academy of Sciences - Technical Sciences 2007, 55, 1, 107-113.

[7] Michalewicz Z., Genetic Algorithms + Data Structures = Evolution Programs, Springer-Verlag, Berlin 1996. 